\title{
CRECIMIENTO RESTRINGIDO POR BALANZA DE PAGOS: MÉXICO 1970-2012
}

\section{BALANCE OF PAYMENTS CONSTRAINED GROWTH: MÉXICO 1970-2012}

José Luis Zamora Ortiz Instituto Politécnico Nacional, México luis.jose.j125@gmail.com
Gerardo Ángeles Castro Instituto Politécnico Nacional, México g.angelescastro@gmail.com

\section{RESUMEN}

Este artículo aplica el modelo de crecimiento restringido por balanza de pagos (MCRBP) a la economía mexicana. Se estima la elasticidad ingreso de la demanda de las importaciones reales y la elasticidad de las importaciones con respecto a la tasa de crecimiento de los precios relativos, ambas de largo plazo, y el parámetro de velocidad de ajuste de la ecuación de demanda de importaciones. Se hace uso de la técnica de cointegración del enfoque econométrico conocido como modelo autorregresivo con rezagos distribuidos (ARDL, por sus siglas en inglés) para estimar los parámetros. Mediante un vector de corrección de error se exploran los ajustes de corto plazo. Se encontró que la tasa de crecimiento promedio pronosticada por el MCRBP es muy similar a la tasa de crecimiento promedio verdadera a lo largo del periodo de estudio 1970-2012. 
Palabras clave: restricción de balanza de pagos, elasticidad ingreso de la demanda de las importaciones, crecimiento económico, economía mexicana

Clasificación JEL: C22, F14, F32, F43.

\begin{abstract}
This study applies the balance of payments constrained growth model to mexican economy. The long-run income elasticity of demand for real imports, the long-run elasticity of imports with respect to growth rate of relative price, and the speed of adjustment parameter of import demand equation are estimated. ARDL's cointegration technique is employed to estimate the parameters. Short-run adjustments are explored within a vector error correction framework. The average growth rate predicted by the balance of payments constrained growth model is found to be close to the actual average growth rate over the period 1970-2012.
\end{abstract}

Keywords: Balance-of-payments constraint, income elasticity of demand for imports, economic growth, mexican economy 


\section{INTRODUCCIÓN}

El modelo de crecimiento restringido por balanza de pagos (MCRBP) propuesto por Thirlwall (1979) fue desarrollado con la finalidad de analizar la restricción impuesta a las economías de los países, quienes tienen la necesidad de generar divisas para financiar sus importaciones de bienes y servicios. El modelo proporciona una explicación, del lado de la demanda, del comportamiento de algunas variables incluidas y relacionadas a la balanza de pagos, cuya evolución pueda llegar a limitar el crecimiento económico. Inicialmente, el MCRBP no incluía flujos de capital, posteriormente, Thirlwall y Hussain (1982) agregaron esta variable al modelo original.

El MCRBP ha sido capaz de proporcionar una explicación adecuada a la desaceleración del crecimiento de la economía mexicana tras la liberalización comercial a finales de los ochenta. Moreno-Brid (1998) utilizó por primera vez el MCRBP para el caso de la economía mexicana, en su versión original. A partir de entonces, con el uso de distintas metodologías econométricas se ha analizado la influencia de la restricción de balanza de pagos sobre el crecimiento económico de largo plazo de la economía mexicana: Moreno-Brid (1998, 1999, 2002a y 2003), López y Cruz (2000), Guerrero de Lizardi (2003, 2006), Ocegueda (2000), Loría (2001), Rocha y Tadeu (2010), Bagnai (2010), todos ellos han llegado a la conclusión de que se cumple la ley de Thirlwall para la economía mexicana.

Estudios realizados por autores como Moreno-Brid (1999, 2002b), López y Cruz (2000), Guerrero de Lizardi (2003, 2006), Pacheco-López y Thirlwall (2004), Pacheco-López (2005) y Cardero y Galindo (2005) coinciden en que la elasticidad ingreso de la demanda de las importaciones mexicanas se ha incrementado de manera significativa a partir de la liberalización comercial a mediados de los años ochenta; sin que tal fenómeno sea compensado por un mayor crecimiento de las exportaciones. Lo anterior implica, de acuerdo con el MCRBP, una reducción en la tasa de crecimiento económico consistente con el equilibrio de la balanza de pagos, es decir, una tasa de crecimiento lo bastante baja para prevenir el incremento de déficits comerciales crónicos ocasionados por un mayor crecimiento de las importaciones en relación con el aumento de las exportaciones. Sin embargo, Ibarra (2011a, 2011b) encontró que la elasticidad ingreso de la demanda de las 
importaciones de bienes intermedios no aumentó de manera significativa después de la apertura comercial.

Este artículo prueba el MCRBP para el caso de la economía mexicana, se basa en varios estudios recientes sobre el uso de técnicas de cointegración para estudiar la restricción de largo plazo impuesta por los requerimientos de divisas necesarias para el crecimiento económico. El periodo analizado es 1970-2012. Se utilizó un modelo autorregresivo con rezagos distribuidos (ARDL, por sus siglas en inglés) en el que se exploraron las relaciones de cointegración entre las variables.

El resto del documento está organizado de la siguiente manera. La sección dos desarrolla el modelo teórico básico de la ley del crecimiento de Thirlwall; la tres plantea el modelo econométrico utilizado, la cuatro presenta los resultados obtenidos y la sección cinco concluye con una discusión de los resultados.

\section{LA LEY DEL CRECIMIENTO DE THIRLWALL}

Thirlwall afirma que las restricciones de demanda son válidas para la mayor parte de los países y que actúan antes que las restricciones de oferta. Consecuentemente, para una economía abierta, la demanda constituye la principal restricción al crecimiento y, por lo tanto, el desempeño económico estará sujeto al comportamiento de la balanza de pagos. La principal idea del MCRBP es que un país no puede crecer a una tasa mayor que aquella que es consistente con el equilibrio de su cuenta corriente, puesto que no es posible mantener por un periodo prolongado una balanza de pagos deficitaria; necesariamente tiene que ser financiada por entradas de capital de corto plazo, lo que conduce a un incremento de la relación deuda externa neta sobre producto interno bruto (PIB). Si un país intenta financiar su déficit de manera prolongada a través de entradas de capital externo, los mercados financieros a nivel internacional presionarán a la moneda nacional, con lo que se crean las condiciones para que su tipo de cambio colapse, es decir, habría un escenario de depreciación e inflación. Por lo tanto, la tasa de crecimiento de cualquier economía, en el largo plazo, debe ser aquella que es consistente con el equilibrio de su balanza de pagos (McCombie, 2003; Panico, 2003; Thirlwall, 2003). 
El modelo de crecimiento restringido por la balanza de pagos de Thirlwall analiza el efecto de la demanda de exportaciones sobre el crecimiento económico e introduce el fenómeno de restricción de balanza de pagos. La idea fundamental es que, para toda economía abierta, la demanda de exportaciones constituye el principal componente de la demanda autónoma. De tal forma que a largo plazo el crecimiento económico estará basado en el incremento de las exportaciones. Recordemos que las exportaciones afectan de manera directa a la demanda, pero también lo hacen indirectamente, puesto que el consumo y la inversión crecen a mayor velocidad. Por tanto, si consideramos ambos efectos, constatamos que el incremento de las exportaciones (x) determina el crecimiento de la producción (y).

Podemos representar la tasa de crecimiento de las exportaciones como: $\mathrm{x}=\eta\left(\mathrm{P}_{d}-\mathrm{P}_{f}\right)+\varepsilon(\mathrm{Z})$, donde $\mathrm{P}_{d} \mathrm{y}_{f}$ son los precios internos y externos respectivamente, $\mathrm{Z}$ es el ingreso externo, $\varepsilon(>0)$ es la elasticidad ingreso de la demanda de exportaciones y $\eta(<0)$ es la elasticidad precio de la demanda de exportaciones. Los precios internos son considerados como factor endógeno, en tanto que el ingreso y los precios externos son factores exógenos.

Thirlwall (2003) introdujo al estudio del crecimiento económico la restricción de balanza de pagos, lo hizo retomando la ecuación que representa la tasa de crecimiento de las exportaciones, le añadió la ecuación de demanda de importaciones y la condición de equilibrio de la balanza de pagos.

Las ecuaciones de demanda de exportaciones e importaciones quedan definidas respectivamente de la siguiente manera:

$$
\begin{aligned}
& \mathrm{x}=\eta\left(\mathrm{P}_{d}-\mathrm{P}_{f}-e r\right)+\varepsilon(\mathrm{Z}) \\
& \mathrm{m}=\Psi\left(\mathrm{P}_{f}-e r-\mathrm{P}_{d}\right)+\pi(\mathrm{y})
\end{aligned}
$$

Donde $\pi(>0)$ es la elasticidad ingreso de la demanda de importaciones, $\Psi$ representa la elasticidad precio de la demanda de importaciones, er es el tipo de cambio y y es la tasa de crecimiento del PIB. 
La condición de equilibrio en la cuenta corriente, expresada en tasas de cambio, se define como:

$$
\mathrm{P}_{d}+\mathrm{x}=\mathrm{P}_{f}+\mathrm{m}+e r
$$

A través de la sustitución de las ecuaciones de demanda de exportaciones e importaciones al interior de la condición de equilibrio en la cuenta corriente obtenemos la tasa de crecimiento del ingreso nacional que es consistente con el equilibrio de la balanza de pagos:

$$
\mathrm{P}_{d}+\eta\left(\mathrm{P}_{d}-\mathrm{P}_{f}-e r\right)+\varepsilon(\mathrm{Z})=\mathrm{P}_{f}+\Psi\left(\mathrm{P}_{f}-e r-\mathrm{P}_{d}\right)+\pi(\mathrm{y})+e r
$$

Si se transforma algebraicamente la ecuación (4) es posible encontrar una expresión que representa el crecimiento del PIB que es consistente con el equilibrio de la cuenta corriente:

$$
\mathrm{y}=\left((1+\eta+\Psi)\left(\mathrm{P}_{d}-\mathrm{P}_{f}-e r\right)+\varepsilon(\mathrm{z})\right) / \pi
$$

De acuerdo con Thirlwall (2003), la ecuación (5) expresa lo siguiente:

a) una mejora en los términos de intercambio $\left(\mathrm{P}_{d}-\mathrm{P}_{f}-e r\right)>0$ tiene el potencial de restablecer la tasa de crecimiento que es consistente con el equilibrio de la balanza de pagos;

b) si los precios internos aumentan en mayor proporción que los externos provocará una disminución de la tasa de crecimiento que es consistente con el equilibrio de la balanza de pagos, esto es, si la suma de las elasticidades precio negativas es mayor que uno: $(1+\eta+\Psi)<0$;

c) una depreciación de la moneda (er $>0)$ tendrá la capacidad de aumentar la tasa de crecimiento que es consistente con el equilibrio de la balanza de pagos si la suma de las elasticidades precio es mayor que uno.

d) el crecimiento económico del país (y) está vinculado al crecimiento de otros países (z), por esta razón, la tasa de crecimiento que un país puede alcanzar, preservando el equilibrio en su balanza de pagos, dependerá de su elasticidad ingreso de demanda de exportaciones $(\varepsilon)$. 
e) la tasa de crecimiento económico de un país que es consistente con el equilibrio de su balanza de pagos se encuentra inversamente relacionada con su demanda de importaciones.

Al suponer que los precios relativos ${ }^{1}$ permanecen sin cambio ${ }^{2}$ entonces la tasa de crecimiento consistente con la balanza de pagos se transforma:

$$
Y_{t}^{*}=\varepsilon\left(z_{t}\right) / \pi
$$

Si tomamos como variable proxy de $\mathrm{z}$ a la tasa de crecimiento de las exportaciones $\mathrm{x}$, esto conduce a que la ecuación anterior quede de la siguiente manera:

$$
Y_{t}^{*}=x_{t} / \pi
$$

A la ecuación (7) se le conoce como ley del crecimiento de Thirlwall, la cual menciona que la tasa de crecimiento económico de cualquier economía en el largo plazo se hallará restringida por el equilibrio en la cuenta corriente de su balanza de pagos.

1 A la relación del precio de un bien o servicio con el de otro se le denomina precio relativo. El precio relativo del bien $\mathrm{X}$ respecto del bien $\mathrm{Y}$, implica la relación del precio del bien $\mathrm{X}$ con el precio del bien $\mathrm{Y}$; es decir: $\mathrm{Px}, \mathrm{y}=\mathrm{Px} / \mathrm{Py}$. Esta razón señala la cantidad de unidades del bien Y que es necesario dejar de consumir para obtener una unidad más del bien X. Un precio relativo es un costo de oportunidad.

2 Si los precios relativos permanecen sin cambio en el corto plazo la balanza comercial no se modifica. Sin embargo, en un escenario de balanza comercial deficitaria, una depreciación puede conducir al equilibrio o incluso al superávit de la siguiente manera: con la depreciación se paga una mayor cantidad de moneda nacional por moneda extranjera, con lo que se incrementa el tipo de cambio nominal, en consecuencia también aumentan los precios relativos. Las importaciones aumentan su precio en moneda nacional, por lo que su demanda disminuye; por otra parte, el precio de las exportaciones en moneda extranjera baja y aumenta su demanda. El resultado es que, en el caso de que se cumpla la condición Marshall-Lerner, aumentan las exportaciones netas lo que mejora la balanza comercial. Una apreciación de la moneda nacional disminuye los precios relativos, lo que lleva a que la demanda de importaciones aumente y las exportaciones disminuyan, esto conduce a un deterioro de la balanza comercial.

En suma, una depreciación hace que las importaciones sean menos competitivas al volver los precios relativos en contra de las importaciones y a favor de los productos nacionales; mientras que una apreciación hace que los precios relativos se muevan a favor de las importaciones y en contra de la producción interior. 


\section{MODELO ECONOMÉTRICO}

Generalmente se comprueba la ley de Thirlwall mediante la comparación de las tasas de crecimiento efectivas del producto real con las pronosticadas por la ecuación (7); donde la elasticidad ingreso de la demanda de las importaciones determina en gran medida el resultado. Al expresar las variables de la relación de largo plazo de la función de demanda de importaciones en logaritmos tenemos:

$$
L M_{t}=\alpha+\pi L Y_{t}+\beta L P R_{t}+\omega_{t}
$$

donde $L M_{t}$ es el logaritmo de las importaciones reales, $L Y_{t}$ es el logaritmo del ingreso real, $L P R_{t}$ es el logaritmo de los precios relativos (se encuentra definido como la razón de los precios nacionales a precios externos) y $\omega_{t}$ es el término de error.

Avances recientes en econometría proponen que a la relación de largo plazo en la ecuación (8) se le debe incorporar el proceso de ajuste dinámico de corto plazo. Con esta finalidad reexpresamos la ecuación (8) como un modelo de corrección de error, tal como lo sugieren Pesaran, Shin y Smith (2001):

$$
\begin{aligned}
& \Delta L M_{t}=b_{0}+\sum_{\mathrm{i}=0}^{\mathrm{m} 1} b_{1 i} \Delta \mathrm{LM}_{t-i}+\sum_{\mathrm{i}=0}^{\mathrm{m} 2} b_{2 i} \Delta \mathrm{LY}_{t-i}+\sum_{\mathrm{i}=0}^{\mathrm{m} 3} b_{3 i} \Delta \mathrm{LPR}_{t-i}+ \\
& +b_{4} L M_{t-i}+b_{5} L Y_{t-i}+b_{6} L P R_{t-i}+\mathrm{v}_{t}
\end{aligned}
$$

Este enfoque es conocido como modelo autorregresivo con rezagos distribuidos (ARDL, por sus siglas en inglés). Una característica importante de dichos modelos es que, comúnmente, producen estimaciones insesgadas de los coeficientes de largo plazo, a pesar de que algunas de las variables explicativas sean endógenas (Pesaran y Shin, 1999). Otra ventaja es que con la estimación por medio del enfoque de pruebas de límites es posible utilizar variables integradas de orden uno I(1) y cero I(0) en la misma ecuación; lo que no es posible en el enfoque de Johansen, ya que para 
construir un modelo de vector de corrección de errores es necesario que las variables tengan el mismo orden de integración.

Los modelos ARDL en forma de corrección de errores proporcionan los coeficientes de largo y de corto plazo de manera simultánea. Los efectos de corto plazo se reflejan a través de las estimaciones de los coeficientes que provienen de las variables en primeras diferencias. Los efectos de largo plazo provienen de las estimaciones de $b_{5}$ y $b_{6}$, los cuales son normalizados sobre $b_{4}$. También incluye el coeficiente de velocidad de ajuste, que en un modelo de corrección de errores corresponde al coeficiente del error rezagado de largo plazo. Con la finalidad de determinar si el ajuste de las variables tiende a un valor de equilibrio de largo plazo se utilizan las estimaciones de $b_{4}, b_{5}$ y $b_{6}$ para construir un término de corrección de error (TCE), el cual sustituye a las variables en niveles rezagadas de la ecuación (9), con lo que obtenemos:

$$
\begin{aligned}
& \Delta L M_{t}=\mathrm{c}_{0}+\sum_{\mathrm{i}=0}^{\mathrm{m} 1} \mathrm{c}_{1 i} \Delta \mathrm{LM}_{t-i}+\sum_{\mathrm{i}=0}^{\mathrm{m} 2} \mathrm{c}_{2 i} \Delta \mathrm{LM}_{t-i}+\sum_{\mathrm{i}=0}^{\mathrm{m} 3} \mathrm{c}_{3 i} \Delta \mathrm{LM}_{t-i}+ \\
& +\lambda T C E_{t-i}+\mu_{t}
\end{aligned}
$$

En la ecuación (10) $\lambda$ representa el parámetro de velocidad de ajuste. Se estima nuevamente esta ecuación teniendo en cuenta los mismos rezagos utilizados anteriormente; si el coeficiente obtenido de $T C E_{t-i}$ es negativo y significativo indica, una vez más, la existencia de cointegración entre las variables y el hallazgo de una tendencia de largo plazo hacia el equilibrio ${ }^{3}$.

$3 \quad$ El hecho de que tenga signo negativo y sea significativo indica que la variable dependiente se mueve en el tiempo hacia su nivel de equilibrio de largo plazo. 


\section{RESULTADOS}

En este artículo se utilizaron series de datos anuales durante el periodo 1970-2012 ${ }^{4}$. Las variables de la ecuación (8) cubrieron el requisito de ser a lo mucho I(1), recordemos que si alguna fuera $\mathrm{I}(2)$, los resultados provenientes del procedimiento de cointegración de Pesaran, Shin y Smith (2001) no serían consistentes y eficientes.

Se aplicaron a las series las pruebas de raíces unitarias: augmented Dickey-Fuller (ADF) (1979, 1981), Phillips-Perron (PP) (1988) y ElliottRothenberg-Stock (ERS) (1996), con lo que se verificó que son estacionarias, ya sea en niveles o en primeras diferencias. En el cuadro 1 se observa que las variables son integradas de orden 1 o 0 , pero no superior, lo que valida la instrumentación del enfoque de pruebas de límites sugerido por Pesaran, Shin y Smith (2001).

Cuadro 1. Pruebas de raíz unitaria

\begin{tabular}{|l|l|l|l|}
\hline Variables & ADF & PP & ERS \\
\hline LMt & 0.2146 & 0.4077 & $5.693846^{*}$ \\
\hline LYt & 0.0801 & 0.2491 & 28.70061 \\
\hline LPRt & 0.5907 & 0.5907 & 16.47537 \\
\hline$\Delta$ LMt & $0.0002^{* *}$ & $0.0002^{* *}$ & $1.488815^{* *}$ \\
\hline $\boldsymbol{\Delta L Y t}$ & $0.0004^{* *}$ & $0.0004^{* *}$ & $1.239741^{* *}$ \\
\hline $\boldsymbol{\Delta L P R t}$ & $0.0001^{* *}$ & $0.0001^{* *}$ & $1.175878^{* *}$ \\
\hline
\end{tabular}

Notas: Las regresiones de las muestras en niveles incluyen constante y tendencia determinista. Las regresiones de las muestras en primeras diferencias son con constante y sin tendencia. * Indica rechazo de la hipótesis nula: presencia de raíz unitaria a 5\% de significancia. ** Indica rechazo de la hipótesis nula: presencia de raíz unitaria a $1 \%$ de significancia. $\Delta$ representa primera diferencia.

Con la finalidad de verificar si existe una relación de cointegración de largo plazo entre las variables de la ecuación (8) se realizó una estimación en dos etapas. En primer lugar, se comprobó la suficiencia estadística del modelo. Por lo que se procedió a encontrar el número óptimo de rezagos de las variables en primera diferencia (se utilizó el criterio de información de Hannan-Quinn, Schwarz y Akaike) y se comprobó que el modelo cumple con las pruebas de diagnóstico convencionales. Después de que

4 La definición y fuente de los datos se encuentra en el apéndice. 
se confirmó la suficiencia estadística del modelo, en una segunda etapa se procedió a verificar la existencia de cointegración.

Pesaran, Shin y Smith (2001) proponen dos pruebas de cointegración. En la primera proporcionan valores críticos para la prueba t correspondiente, con límites superiores e inferiores, que están en función de si las variables de la ecuación son todas integradas de orden 0 (límite inferior) o de 1 (límite superior). Se comprueba que existe cointegración cuando el valor absoluto del estadístico sobrepasa el límite superior. Proponen, en segundo lugar, una prueba $\mathrm{F}$ para la significancia de las variables en nivel, bajo la hipótesis nula de que la velocidad de ajuste y los coeficientes de la ecuación (9) son conjuntamente iguales a cero. En Pesaran, Shin y Smith (2001) se encuentran los valores críticos superiores e inferiores para estas pruebas, se acepta que existe cointegración cuando los estadísticos $\mathrm{F}$ y $\mathrm{t}$ son mayores en valor absoluto que el límite superior (ver cuadro 2).

Cuadro 2. Pruebas de límites. Relación de largo plazo supuesta: F/t (LM I LY, LPR)

\begin{tabular}{|l|c|c|c|c|}
\hline & $95 \%$ LI & 95\% LS & $\mathbf{9 7 . 5 \% ~ L I ~}$ & $\mathbf{9 7 . 5 \% ~ L S ~}$ \\
\hline Estadístico F & & & & \\
\hline $\mathbf{5 . 7 9}$ & 3.79 & 4.85 & 4.41 & 5.52 \\
\hline Estadístico t & & & & \\
\hline $\mathbf{- 3 . 9 8}$ & -2.86 & -3.53 & -3.13 & -3.80 \\
\hline
\end{tabular}

Notas: Los estadísticos de prueba t y F se ubican arriba del límite superior a los niveles de significancia de 5\% y de 2.5\% (valores tomados de Pesaran, Shin y Smith (2001), cuadros CI(iii) y CII(iii). La hipótesis nula es: no existe cointegración entre las variables, importaciones, PIB y precios relativos. Las pruebas de límites indican que se puede rechazar la hipótesis nula, lo que implica que existe cointegración entre las variables en estudio.

El modelo ARDL del periodo 1970-2012 fue estimado con mínimos cuadrados ordinarios. Los coeficientes de largo y de corto plazo aparecen en los cuadros 3 y 5 , respectivamente, los coeficientes que son estadísticamente significativos tienen el signo esperado. Correspondientes pruebas estadísticas se encuentran en los cuadros 4 y $6^{5}$, e indican que no existen problemas de normalidad, autocorrelación, heteroscedasticidad, o de incorrecta especificación.

$5 \quad$ A los modelos estimados se les realizaron las pruebas de normalidad White y ARCH para heteroscedasticidad, asimismo se llevó a cabo la prueba de Breusch-Godfrey para probar correlación serial y la prueba de Ramsey para verificar correcta especificación. Este conjunto de pruebas permitió comprobar que no existen dichos problemas a $5 \%$ de significancia. Las pruebas pueden ser enviadas a quien así lo requiera. 
Cuadro 3. Resultados de cointegración por ARDL para la ecuación de demanda de importaciones de largo plazo. Variable dependiente: LMt

\begin{tabular}{|c|c|c|}
\hline Regresores & Coeficiente & Probabilidad \\
\hline Constante & -17.82 & 0.00 \\
\hline LYt & 2.17 & 0.00 \\
\hline LPRt & 1.00 & 0.00 \\
\hline
\end{tabular}

Fuente: elaboración propia con base en resultados econométricos.

Cuadro 4. Pruebas estadísticas en la ecuación de demanda de importaciones de largo plazo

\begin{tabular}{|l|l|l|}
\hline R2 & 0.96 & \\
\hline RSS & 0.11 & \\
\hline Estadístico F & 131.24 & $0.00^{*}$ \\
\hline Estadístico DW & 1.93 & \\
\hline Breusch-Godfrey LM (2) & 2.08 & $0.14^{*}$ \\
\hline Jarque-Bera & 2.52 & $0.28^{*}$ \\
\hline Breusch-Pagan-Godfrey & 2.14 & $0.17^{*}$ \\
\hline Harvey & 1.90 & $0.10^{*}$ \\
\hline Arch (5) & 2.23 & $0.07^{*}$ \\
\hline White & 1.65 & $0.14^{*}$ \\
\hline RESET (1) & 2.14 & $0.07^{*}$ \\
\hline
\end{tabular}

Notas: RSS indica suma de residuos al cuadrado $\mathrm{y}$ * valor de la probabilidad. Fuente: elaboración propia con base en resultados econométricos.

Podemos observar en el cuadro 3 que la elasticidad de largo plazo de las importaciones con respecto al ingreso es de 2.17 , lo que implica que cuando la tasa de crecimiento del PIB real aumenta $1 \%$ la tasa de crecimiento de las importaciones reales se incrementará 2.17 por ciento. Un resultado muy similar es reportado por Moreno-Brid (2003), él encuentra que el valor de equilibrio hipotético de la elasticidad ingreso de la demanda de importaciones para la economía mexicana durante el periodo 1967-1999 es de 2.18, al utilizar la versión original de la ley de Thirlwall; Guerrero de Lizardi (2003) mediante una especificación estocástica descubre que el valor de la elasticidad ingreso de la demanda de las importaciones para los periodos $1940-2000$ y $1982-2000$ es de 1.61 y 2.47, respectivamente. Pacheco-López (2005) utiliza una metodología econométrica similar a la de este artículo y encuentra que la elasticidad ingreso de la demanda de importaciones se incrementó de 1.21 durante el periodo $1973-1987$ a 3.15 para 1985-1999. Las 
diferencias en el valor de la elasticidad pudieron ser ocasionadas por haber utilizado especificaciones econométricas e intervalos de tiempo distintos. La elasticidad de largo plazo de las importaciones con respecto a la tasa de crecimiento de los precios relativos es de 1.00, esto indica que, cuando la tasa de crecimiento de los precios relativos aumenta $1 \%$, la tasa de crecimiento de las importaciones reales se incrementará también en 1 por ciento ${ }^{6}$. La velocidad de ajuste es de -0.17 , significa que cuando la ecuación de demanda de las importaciones se encuentra fuera de su nivel de equilibrio, se ajustará $17 \%$ cada año; en otras palabras, le tomará más de cinco años la convergencia total hacia su nivel de equilibrio.

Cuadro 5. Resultados de cointegración por ARDL para la ecuación de demanda de importaciones de corto plazo. Variable dependiente: $\Delta \mathrm{LMt}$

\begin{tabular}{|c|c|c|}
\hline Regresores & Coeficiente & Probabilidad \\
\hline Constante & -0.01 & 0.13 \\
\hline$\Delta$ LYt & 2.64 & 0.00 \\
\hline$\Delta$ LYt -1 & -0.38 & 0.20 \\
\hline$\Delta$ LPRt & 0.76 & 0.00 \\
\hline$\Delta$ LPRt-1 & 0.15 & 0.10 \\
\hline TCEt-1 & -0.17 & 0.04 \\
\hline
\end{tabular}

Fuente: elaboración propia con base en resultados econométricos.

6 Este es un resultado un tanto anómalo, se esperaría que ante un crecimiento de los precios relativos las importaciones reales disminuyeran. Sin embargo, para la economía mexicana, Ibarra (2011b) reporta un coeficiente positivo para el tipo de cambio real en la ecuación de importaciones. Este hecho puede ser explicado porque ante una depreciación de la moneda las empresas se preparan para una futura expansión de las exportaciones, de esta manera demandan una mayor cantidad de importaciones intermedias de lo que requiere la producción actual. Asimismo, puede ser que la depreciación acelera las exportaciones, lo que impulsa a las empresas a intensificar el uso de importaciones para responder rápidamente a la expansión del mercado. 
Cuadro 6. Pruebas estadísticas en la ecuación de demanda de importaciones de corto plazo

\begin{tabular}{|l|l|l|}
\hline R2 & 0.94 & \\
\hline RSS & 0.04 & \\
\hline Estadístico F & 63.27 & $0.00^{*}$ \\
\hline Estadístico DW & 2.11 & \\
\hline Breusch-Godfrey LM (2) & 1.29 & $0.28^{*}$ \\
\hline Jarque-Bera & 2.31 & $0.31 *$ \\
\hline Breusch-Pagan-Godfrey & 0.41 & $0.93 *$ \\
\hline Glejser & 0.59 & $0.81^{*}$ \\
\hline Arch (1) & 0.15 & $0.69 *$ \\
\hline White & 0.41 & $0.93^{*}$ \\
\hline RESET (1) & 1.54 & $0.22^{*}$ \\
\hline
\end{tabular}

Notas: RSS indica suma de residuos al cuadrado $\mathrm{y} *$ valor de la probabilidad. Fuente: elaboración propia con base en resultados econométricos.

Se utilizaron periodos traslapados de quince años, tal como lo realizó Atesoglu (1993a, 1993b, 1994), para verificar la validez de la ley de Thirlwall para la economía mexicana durante el periodo 1970-2012. Se toman periodos largos debido a la relación de largo plazo establecida por la ley de Thirlwall entre la balanza de pagos y la tasa de crecimiento del PIB. En consecuencia se utilizaron las tasas de crecimiento promedio de la demanda de exportaciones efectiva y del PIB real efectivo en periodos de quince años, se inicia con 19711985 y se finaliza en 1998-2012. La predicción del crecimiento del ingreso real restringido por la balanza de pagos $Y_{t}^{*}$ se obtuvo de la ecuación (7) con la estimación de la elasticidad ingreso de la demanda de importaciones ARDL de largo plazo, 2.17. Los resultados se presentan en el cuadro 7, donde podemos observar que el promedio de la tasa de crecimiento del PIB efectivo es muy parecido al promedio de la tasa de crecimiento del PIB pronosticado. Sin embargo, existen periodos donde la diferencia entre las tasas de crecimiento pronosticadas y efectivas del PIB es amplia. Por ejemplo, periodos que se ubican al principio del cuadro 7, como 1971-1985, 1972-1986, 1973-1987, 1974-1988, y los que se aparecen al final, 1995-2009, 1996-2010, 1997-2011, 1998-2012, parecen sugerir que la tasa de crecimiento del PIB real efectiva y pronosticada se mueven hacia un nivel de convergencia. No obstante, esto no ocurre si observamos los periodos 1981-1995, 1982-1996, 1983-1997 y 1986-2000, puesto que se alejan de cierta convergencia. Se acentúa con mayor profundidad esta divergencia para los años en que la economía mexicana 
registró altos niveles de inflación, específicamente durante el periodo 19821988. Lo que dio como resultado que el crecimiento económico real de México estuviera por debajo de su potencial.

Cuadro 7. Tasas de crecimiento promedio en porcentaje anual, restringida por balanza de pagos y efectiva, 1970-2012

\begin{tabular}{|c|c|c|c|c|c|}
\hline Periodos & $\begin{array}{c}\text { Elasticidad } \\
\text { ingreso de la } \\
\text { demanda de } \\
\text { importaciones } \\
\text { ARDL de largo } \\
\text { plazo } \boldsymbol{\pi}\end{array}$ & $\begin{array}{c}\text { Tasa de } \\
\text { crecimiento } \\
\text { efectiva de } \\
\mathbf{X}\end{array}$ & $\begin{array}{c}\text { Tasa de } \\
\text { crecimiento } \\
\text { efectiva } \\
\text { de Y }\end{array}$ & $\begin{array}{c}\text { Tasa de } \\
\text { crecimiento } \\
\text { pronosticada } \\
\text { de Y* }\end{array}$ & $\begin{array}{c}\text { Diferencia } \\
\text { Y*-Y }\end{array}$ \\
\hline $1971-1985$ & 2.17 & 8.97 & 5.08 & 4.13 & -0.94 \\
\hline $1972-1986$ & 2.17 & 9.08 & 4.56 & 4.18 & -0.37 \\
\hline $1973-1987$ & 2.17 & 8.62 & 4.12 & 3.97 & -0.14 \\
\hline $1974-1988$ & 2.17 & 8.09 & 3.64 & 3.73 & 0.08 \\
\hline $1975-1989$ & 2.17 & 8.45 & 3.52 & 3.89 & 0.37 \\
\hline $1976-1990$ & 2.17 & 9.40 & 3.48 & 4.33 & 0.85 \\
\hline $1977-1991$ & 2.17 & 8.63 & 3.48 & 3.98 & 0.49 \\
\hline $1978-1992$ & 2.17 & 7.99 & 3.49 & 3.68 & 0.18 \\
\hline $1979-1993$ & 2.17 & 7.75 & 3.07 & 3.57 & 0.50 \\
\hline $1980-1994$ & 2.17 & 8.13 & 2.75 & 3.74 & 0.99 \\
\hline $1981-1995$ & 2.17 & 9.74 & 1.78 & 4.48 & 2.70 \\
\hline $1982-1996$ & 2.17 & 10.18 & 1.54 & 4.69 & 3.14 \\
\hline $1983-1997$ & 2.17 & 9.44 & 2.04 & 4.35 & 2.30 \\
\hline $1984-1998$ & 2.17 & 9.35 & 2.66 & 4.30 & 1.64 \\
\hline $1985-1999$ & 2.17 & 9.79 & 2.67 & 4.51 & 1.83 \\
\hline $1986-2000$ & 2.17 & 11.18 & 2.93 & 5.15 & 2.22 \\
\hline $1987-2001$ & 2.17 & 10.56 & 3.17 & 4.86 & 1.69 \\
\hline $1988-2002$ & 2.17 & 10.03 & 3.10 & 4.62 & 1.51 \\
\hline $1989-2003$ & 2.17 & 9.82 & 3.11 & 4.52 & 1.41 \\
\hline $1990-2004$ & 2.17 & 10.05 & 3.11 & 4.63 & 1.51 \\
\hline $1991-2005$ & 2.17 & 10.08 & 2.98 & 4.64 & 1.66 \\
\hline $1992-2006$ & 2.17 & 10.25 & 3.03 & 4.72 & 1.69 \\
\hline $1993-2007$ & 2.17 & 10.16 & 3.00 & 4.68 & 1.67 \\
\hline $1994-2008$ & 2.17 & 9.53 & 2.96 & 4.39 & 1.42 \\
\hline $1995-2009$ & 2.17 & 7.56 & 2.35 & 3.48 & 1.12 \\
\hline $1996-2010$ & 2.17 & 6.92 & 3.11 & 3.19 & 0.07 \\
\hline $1997-2011$ & 2.17 & 6.25 & 3.02 & 2.88 & -0.14 \\
\hline $1998-2012$ & 2.17 & 5.93 & 2.84 & 2.73 & -0.10 \\
\hline
\end{tabular}

Fuente: elaboración propia con datos de la OCDE y con resultados econométricos. 
Se observa que en los tres primeros periodos 1971-1985, 1972-1986 y 1973-1987, igual que en los dos últimos, 1997-2011 y 1998-2012, el promedio de la tasa de crecimiento verdadera de la economía mexicana se encuentra ligeramente por arriba del valor pronosticado. Durante estos periodos la economía mexicana creció por arriba de su potencial de balanza de pagos, lo que es consistente con los déficits de balanza de pagos observados durante la mayor parte de aquellos años.

Asociado con las reformas estructurales que promovieron la apertura comercial y financiera desde mediados de los años ochenta (Pacheco-López, 2005), en los periodos que finalizan en 1988 hasta 2010 el crecimiento verdadero promedio cayó por debajo del potencial de balanza de pagos.

Para el periodo de estudio 1970-2012 la tasa de crecimiento pronosticada del PIB en promedio es de $4.14 \%$ anual, dicho valor se aproxima de manera muy estrecha a la verdadera tasa de crecimiento promedio del PIB real, que fue de $3.60 \%$ anual. Este hallazgo coincide con los trabajos de Razmi (2005), que estudió el caso de la India, de Rocha y Tadeu (2010) y Blecker e Ibarra (2013) quienes analizaron el caso de la economía mexicana; ellos encontraron que el modelo de crecimiento restringido por la balanza de pagos replica con bastante exactitud las tasas de crecimiento promedio verdaderas cuando se consideran periodos muy amplios, de alrededor de medio siglo.

Finalmente, observemos que, en veintitrés de los veintiocho periodos seleccionados de quince años, la tasa de crecimiento pronosticada es mayor que la tasa de crecimiento efectiva del PIB (sólo en 5 periodos ocurrió lo contrario), lo que nos indica clara evidencia de restricción impuesta a la economía mexicana a través de la balanza de pagos. No obstante, lo más lamentable es que dicha restricción aumenta conforme transcurre el tiempo, por ejemplo: en el primer periodo, 1971-1985, la tasa pronosticada del PIB fue de 4.13, para el último periodo, 1998-2012, fue de 2.73, y hay que tener en cuenta que en la mayor parte de los periodos la tasa de crecimiento efectiva sigue muy de cerca a la tasa de crecimiento pronosticada del PIB. En conclusión, los resultados del cuadro 7 indican que la ley de Thirlwall se mantiene y se está profundizando para la economía mexicana. 


\section{CONCLUSIONES}

El propósito de este artículo fue poner a prueba la validez de la hipótesis del MCRBP para la economía mexicana durante el periodo 19702012. Después de una exploración de las propiedades econométricas de las series de datos y con base en la técnica de cointegración conocida como modelo autorregresivo con rezagos distribuidos (ARDL) se encuentra suficiente evidencia para afirmar que existe una relación de largo plazo estable entre las importaciones reales, el PIB real y los precios relativos. Se ha utilizado el vector de cointegración en una estructura de modelo corrector de error para estudiar la dinámica de la relación de corto plazo. Los valores estimados de las elasticidades de largo plazo de la demanda de las importaciones reales con respecto al ingreso real es de $2.17 \mathrm{y}$ de las importaciones reales en relación con la tasa de crecimiento de los precios relativos es de 1.00. La elasticidad de la demanda de las importaciones reales con respecto al ingreso real se encuentra dentro del rango de estudios anteriores.

Se encontró que la tasa de crecimiento promedio del MCRBP $(4.14 \%$ anual) es muy cercana a la tasa real de crecimiento promedio (3.60\% anual) de la economía mexicana durante el periodo 1970-2012. Sin embargo, surgieron diferencias sustanciales cuando se consideraron periodos individuales de quince años. Así, nuestro análisis encontró más apoyo para el MCRBP como una hipótesis de largo plazo. Aunque las elasticidades de corto plazo también resultaron ser estadísticamente significativas.

Si se desea que la economía mexicana tenga un mayor crecimiento económico ésta fundamentalmente debe mejorar su restricción de balanza de pagos (aumentar su tasa de crecimiento pronosticada del PIB). Su política económica debe ser diseñada no sólo con la finalidad de hacer sus exportaciones más atractivas, sino también con el propósito de reducir su elasticidad ingreso de la demanda de importaciones; para que una eventual expansión de la demanda interna o externa no se traduzca en dificultades de balanza de pagos. Acciones cuya realización en el corto plazo resulta urgente para evitar que la restricción externa al crecimiento de la economía mexicana se amplíe y se profundice. 


\section{APÉNDICE}

Definición y fuente de los datos

Los datos provienen de los principales agregados económicos de la Organización para la Cooperación y Desarrollo Económicos (OCDE). Se consideró esta fuente de información porque proporciona todas las series de datos anuales utilizadas en este artículo (incluidos los deflactores implícitos del PIB y de las importaciones) e incorpora periodos muy amplios (las series que se utilizan en este artículo cubren el lapso 1970-2012).

y tasa de crecimiento del PIB (producto interno bruto) real, en millones de dólares, $2005=100$.

$\mathbf{x}$ tasa de crecimiento de las exportaciones reales, en millones de dólares, $2005=100$.

LM logaritmo de las importaciones reales, en millones de dólares, $2005=100$.

LY logaritmo del PIB real, en millones de dólares, $2005=100$.

LPR logaritmo de los precios relativos, están definidos como la proporción de precios nacionales a externos. Se utilizó el deflactor implícito del PIB como proxy de los precios internos y el deflactor de precios implícito de las importaciones como proxy de los precios externos. 


\section{REFERENCIAS}

Atesoglu, H.S. 1993a. "Balance of payments constrained growth: Evidence from the United States", Journal of Post Keynesian Economics, 15(4): 507-514.

.1993b. "Exports, capital flows, relative prices, and economic growth in Canada", Journal of Post Keynesian Economics, 16(2): 289-297.

.1994. "Balance of payments determined growth in Germany", Applied Economics Letters, 1(6): 89-101.

Bagnai, A. 2010. "Structural changes, cointegration and the empirics of Thirlwall's law", Applied Economics, 42(10): 1315-1329.

Blecker, R.A. y C.A. Ibarra. 2013. "Trade liberalization and the balance of payments constraint with intermediate imports: The case of Mexico revisited", Structural Change and Economic Dynamics, (25): 33-47.

Cardero, M.E. y L.M. Galindo. 2005. "From the import substitution model to the import-export model: Reassessing Mexico's trade liberalization process during the last two decades", The Journal of Economic Asymmetries, 2(2): 71-97.

Dickey, D.A. y W.A. Fuller. 1979. "Distribution of the estimators for autoregressive time series with a unit root", Journal of the American Statistical Association, 74(366): 427-431.

_.1981. "Likelihood ratio statistics for autoregressive time series with a unit root", Econometrica, 49(4): 1057-1072.

Elliott, G., T.J. Rothenberg y J.H. Stock. 1996. "Efficient tests for an autoregressive unit root", Econometrica, 64(4): 813-836.

Guerrero de Lizardi, C. 2003. "Modelo de crecimiento restringido por balanza de pagos. Evidencia para México 1940-2000”, El Trimestre Económico, (278): 253-273.

_2006. "Determinantes del crecimiento económico en México, 1929-2003: una perspectiva poskeynesiana", Investigación Económica, (225): 127-158.

Ibarra, C.A. 2011a. "A note on intermediate imports and the BPCG model in Mexico", Economic Change and Restructuring, 44(4): 357-368. 
.2011b. Import elasticities and the external constraint in Mexico. Economic Systems, 35(3): 363-377.

López, J. y A. Cruz. 2000. "Thirlwall's law and beyond: the Latin American experience", Journal of Post-Keynesian Economics, 22(3): 477-496.

Loría, E. 2001. "La restricción externa y dinámica al crecimiento de México a través de las propensiones del comercio, 1970-1999”, Estudios Económicos, 16(2): 227-251.

McCombie, J. 2003. "Balance-of-payments-constrained economic growth", en J. E. King (comp.), The Elgar Companion to Post Keynesian Economics, Reino Unido, Edward Elgar, pp. 19-23.

Moreno-Brid, J.C. 1998. "Balance-of-payments constrained economic growth: The case of Mexico", Banca Nazionale del Lavoro Quarterly Review, 51(207): 413-433.

. 1999. "Mexico's economic growth and the balance of payments constraint: A cointegration analysis", International Review of Applied Economics, 13(2): 149-159.

.2002a. "A new approach to test the balance-of-payments-constrained growth model, with reference to the mexican economy", en P. Davidson, (comp.), A Post Keynesian Perspective on 21st Century Economic Problems, Reino Unido, Edward Elgar, pp. 89-107.

.2002b. "Liberalización comercial y la demanda de importaciones en México", Investigación Económica, LXII(240): 13-50. .2003. "Capital flows, interest payments and the balance-of-payments constrained growth model: A theoretical and empirical analysis", Metroeconomica, 54(2-3): 346-365.

Ocegueda, J.M. 2000. "La hipótesis de crecimiento restringido por balanza de pagos. Una evaluación de la economía mexicana 1960-1997”, Investigación Económica, LX(232): 91-122.

Pacheco-López, P. y A. Thirlwall. 2004. "Trade liberalisation in Mexico: Rhetoric and reality", Banca Nazionale del Lavoro Quarterly Review, 57(229): 141-167. 
Pacheco-López, P. 2005. "The effect of trade liberalization on exports, imports, the balance of payments and growth: The case of Mexico", Journal of Post Keynesian Economics, 27(4): 595-619.

Panico, C. 2003. "Growth and income distribution”, en J.E. King (comp.), The Elgar Companion to Post Keynesian Economics, Reino Unido, Edward Elgar, pp. 264-270.

Pesaran, M.H. y Y. Shin. 1999. "An autoregressive distributed-lag modelling approach to cointegration analysis", en S. Strom (comp.), Econometrics and Economic Theory in the 20th Century: The Ragnar Frisch Centennial Symposium, Cambridge, Cambridge University Press, pp. 371-413.

Pesaran, M.H., Y. Shin y R.J. Smith. 2001. "Bounds testing approaches to the analysis of level relationships", Journal of Applied Econometrics, 16(3): 289-326.

Phillips, P. y P. Perron. 1988. "Testing for a unit root in time series regression", Biometrika, 75(2): 335-346.

Razmi, A. 2005. "Balance of payments constrained growth model: the case of India", Journal of Post Keynesian Economics, 27(4): 655-687.

Rocha, R. y G. Tadeu. 2010. "Structural change, balance-of-paymentsconstraint, and economic growth: Evidence from the multisectoral Thirlwall's law", Journal of Post Keynesian Economics, 33(1): 169-204.

Thirlwall, A.P. 1979. "The balance of payments constraint as an explanation of international growth rate differences", Banca Nazionale del Lavoro Quarterly Review, 32(128): 45-53. .2003. La naturaleza del crecimiento económico. Un marco alternativo para comprender el desempeño de las naciones, México, Fondo de Cultura Económica, pp. 128.

Thirlwall, A.P. y M.N. Hussain. 1982. "The balance of payments constraint, capital flows, and growth rate differences between developing countries", Oxford Economic Papers, 34(3): 498-510. 